\title{
Bioinformatics analysis of the network of histone H3 lysine 9 trimethylation in acute myeloid leukaemia
}

\author{
AISHU DONG ${ }^{1 *}$, WENBIN YANG $^{2 *}$, HE HUANG $^{2}$, XIAOHAI ZHOU ${ }^{2}$, \\ ZHEWEI $\mathrm{HE}^{3}$, RONGXING YAO ${ }^{2}$ and WENJIAN GUO ${ }^{2}$ \\ Departments of ${ }^{1}$ Emergency, ${ }^{2}$ Haematology, The Second Affiliated Hospital and \\ Yuying Children's Hospital of Wenzhou Medical University, Wenzhou, Zhejiang 325027; \\ ${ }^{3}$ Second Clinical College of Wenzhou Medical University, Wenzhou, Zhejiang 325000, P.R. China
}

Received January 29, 2020; Accepted May 6, 2020

DOI: $10.3892 /$ or.2020.7627

\begin{abstract}
Changes in histone H3 lysine 9 trimethylation (H3K9me3) may be related to the development of drug-resistant acute myeloid leukaemia (AML); insights into the network of $\mathrm{H} 3 \mathrm{~K} 9$ me3 may improve patient prognosis. Patient data were derived from the Gene Expression Omnibus (GEO) database and data from AML cells treated with chidamide, a novel benzamide chemical class of histone deacetylase inhibitor (HDACi), in vitro were derived from ChIP-seq. Patients and AML cell data were analysed using GEO2R, GOseq, KOBAS, the STRING database and Cytoscape 3.5.1. We identified several genes related to the upregulation or downregulation of $\mathrm{H} 3 \mathrm{~K} 9 \mathrm{me} 3$ in AML patients; some of these genes were related to apoptosis, autophagy, and the pathway of cell longevity. AML cells treated with chidamide in vitro showed the same gene changes. The protein interactions in the network did not have significantly more interactions than expected, suggesting the need for more research to identify these interactions. One compelling result from the protein interaction study was that sirtuin 1 (SIRT1) may have an indirect interaction with lysine-specific demethylase 4A (KDM4A). These results help explain alterations of $\mathrm{H} 3 \mathrm{~K} 9 \mathrm{me} 3$ in $\mathrm{AML}$ that may direct further studies aimed at improving patient prognosis. These results may also provide a basis for chidamide as a treatment strategy for AML patients in the future.
\end{abstract}

Correspondence to: Dr Wenjian Guo, Department of Haematology, The Second Affiliated Hospital and Yuying Children's Hospital of Wenzhou Medical University, 109 College West Road, Wenzhou, Zhejiang 325027, P.R. China

E-mail: pxzc1981@163.com

*Contributed equally

Key words: H3K9me3, acute myeloid leukaemia, bioinformatics analysis

\section{Introduction}

Acute myeloid leukaemia (AML) is one of the most common malignant clonal diseases of the circulatory system; it carries a high mortality rate (including a high treatment-related mortality rate of approximately $1.57 / 100,000$ individuals per year in China) and a high recurrence rate (1). The prognosis of most AML patients is poor. The cure rate of AML patients (except for that of acute promyelocytic leukaemia) under 60 years of age is $35-40 \%$, but this rate is only $5-15 \%$ in patients over 60 years of age in China. Strategies designed to improve prognosis and to improve these high rates are the foci of investigation among researchers.

Histone modification is an epigenetic event related to the prognosis of malignant haematologic diseases; histone deacetylase was recently found to be associated with the prognosis in lymphoma (2). A study reported that loss of function and deletions in zeste homolog 2 (EZH2) [a histone methyltransferase that is responsible for transcriptional repression of target genes by trimethylation of lysine 27 on histone H3 (H3K27me3)] are frequent in myeloid malignancies such as myelodysplastic syndromes (MDS), atypical chronic myelogenous leukaemia (CML), T cell acute lymphoblastic leukaemia (T-ALL) and myelofibrosis; these mutations are generally associated with poorer patient prognosis with reduced overall survival (OS) and event-free survival (3-9). Mixed-lineage leukaemia (MLL)-rearranged leukaemias have distinct clinical features and poor prognosis. The majority of MLL translocations result in oncogenic fusion proteins in which the native methyltransferase domain is replaced with sequences that interact with disruptor of telomeric silencing 1-like (DOT1L) directly or indirectly. MLL-rearranged leukaemia depends on aberrant histone H3 lysine 79 (H3K79) methylation by DOT1L $(10,11)$. Another study showed that H3K9me3 deregulation in AML occurred preferentially as a decrease in histone $\mathrm{H} 3$ lysine 9 trimethylation (H3K9me3) levels at core promoter regions. When the H3K9me3 signature was combined with established clinical prognostic markers, it outperformed prognosis predictions based on clinical parameters alone (12). Taken together, these studies suggest that histone methylation is important for prognosis; nevertheless, there have been no studies of the pathways or regulatory mechanisms of $\mathrm{H} 3 \mathrm{~K} 9 \mathrm{me} 3$ in AML cell lines. 
Previous research from our team found that levels of H3K9me3 could be affected by chidamide, a novel benzamide chemical class of histone deacetylase inhibitor (HDACi), an agent that alters expression levels of sirtuin 1 (SIRT1) (a histone deacetylase), and enhances the cytotoxicity of drugs in AML cells (13). Therefore, we performed the present study to explore the pathway of $\mathrm{H} 3 \mathrm{~K} 9 \mathrm{me} 3$ as well as the regulatory mechanisms of SIRT1 on H3K9me3. This study may also provide effective treatment strategies consequently improving the prognosis of AML patients.

\section{Materials and methods}

Samples and databases. A total of 108 primary AML samples and 36 control samples were selected from the Gene Expression Omnibus (GEO) database [GEO accession no. GSE20452 (12), last update, March 22, 2012]. In GSE20452, blasts from patients with AML were obtained at the time of diagnosis. Two batches of experiments were performed and analysed separately. One group of specimens contained AML samples $(n=38)$ and the other contained AML samples $(n=70)$, CD34 ${ }^{+}$progenitor cells $(n=21)$ and white blood cells $(n=15)$ as controls. We used GEO2R (https://www.ncbi.nlm.nih. gov/geo/geo2r/) to compare two groups of samples so as to identify genes that are differentially expressed across experimental conditions. GEO2R terms with corrected P-values $<0.05$ were considered significant, as were $\log \mathrm{FC}$ of GEO2R terms $>1.0$.

Different peak analysis. Different peak analysis was based on the fold-enrichment of peaks in various experiments. A peak was defined as different when the odds ratio (OR) between two groups was more than 2. Using the same method, genes associated with different peaks were identified and subjected to Gene Ontology (GO) enrichment analysis. We used KEGG Orthology-Based Annotation System (KOBAS) 3.0 online (14) to test the statistical enrichment of peak-related genes in Kyoto Encyclopedia of Genes and Genomes (KEGG) (15-17) pathways. GO terms with corrected P-values $<0.05$ were considered significantly enriched by peak-related genes.

Analysis of protein interactions. The STRING (https://string-db. org/) database provides protein-protein interaction (PPI) information, including direct (physical) and indirect (functional) associations (18). Pathways from KEGG and the extended network were constructed for $\mathrm{m} 1 \mathrm{~A}$ regulators and related protein-coding genes signatures using Cytoscape 3.5.1.

Cell line. The AML cell line THP-1 was kindly donated by Professor Ravi Bhatia (City of Hope National Medical Center, Duarte, CA, USA). The THP-1 cell line was cultured in Iscove's modified Dulbecco's medium or RPMI-1640 medium (Invitrogen; Thermo Fisher Scientific, Inc.) with $10 \%$ foetal bovine serum (FBS) at $37^{\circ} \mathrm{C}$ in a humidified incubator containing $5 \% \mathrm{CO}_{2}$.

Drug treatment. Chidamide was kindly donated by Shenzhen Chipscreen Co. AML cells were treated for 24 h. For THP-1 cells, the dose of chidamide was $0.5 \mu \mathrm{M}$ (20).
Chromatin immunoprecipitation sequencing (ChIP-seq). Chromatin immunoprecipitation (ChIP) experiments were performed as previously described $(13,21)$, and ChIP-seq was based on the Illumina Technology Sequencing platform (Illumina, Inc., USA). The single/paired-end method $(13,21)$ was used to complete the ChIP-seq sequencing analysis of the THP-1 cell line. The antibody against histone was H3K9me3 (cat. \#4260, RRID: AB_10828006; Cell Signaling Technology, Inc.).

Statistical analysis. The significances of differences were calculated using the moderated t-statistic (only available when two groups of samples were defined). P-values were adjusted for multiple testing. Genes with the smallest P-values were considered the most reliable. $\mathrm{P}<0.05$ after adjustment was considered statistically significant. $\log 2$-fold change between two experimental conditions (only available when two groups of samples were defined) was carried out. Moderated F-statistic combined the t-statistics was conducted for all pair-wise comparisons into an overall test of significance for that gene (only available when more than two groups of samples are defined).

\section{Results}

Differential expression of genes across experimental conditions between AML and control samples. When samples were collected from the GEO database (accession no. GSE20452), the differential expression of genes between AML samples and control samples was analysed using GEO2R. There were more than 2,000 genes that showed significant differential expression. The definition of the value in $\log (\mathrm{FC})$ for upregulation was $>1.0$, and the definition of the value in $\log (\mathrm{FC})$ for downregulation was $<-1.0$. According to the definition of upregulation or downregulation together with $\mathrm{P}<0.05$, there were 147 genes related to alterations in $\mathrm{H} 3 \mathrm{~K} 9 \mathrm{me} 3$ showing downregulation and 170 genes related to the change in H3K9me3 showing upregulation (data not shown).

Function and network analysis. The function of genes collected from GEO were further analysed using geometric mean of semantic similarities in biological processes (BPs), molecular functions (MFs), and cellular components (CCs), that were assessed using the GOSemSim package (22) by considering the GO topological structure in a more precise and unbiased manner. In BPs, there were 20 processes that showed significant changes (Fig. 1A). The main processes were related to metabolism, including cofactor metabolic process, cellular metabolic processes, and others. In MFs, there were only three significantly altered processes: Protein binding, binding, and molecular-function (Fig. 1B). In CCs, there were 20 processes that showed significant alterations, including intracellular and extracellular processes (Fig. 1C). The relationship of GO items in the regulation of apoptosis and autophagy are shown in Fig. 2A and B. These GO items were included in BPs, MFs, and CCs.

The related pathways were predicted using KOBAS 3.0 online. One of these pathways, the longevity pathway (Fig. 3), may related to the survival of leukaemia cells, including the PI3K/AKT pathway, the AMPK pathway, the FOXO 
A

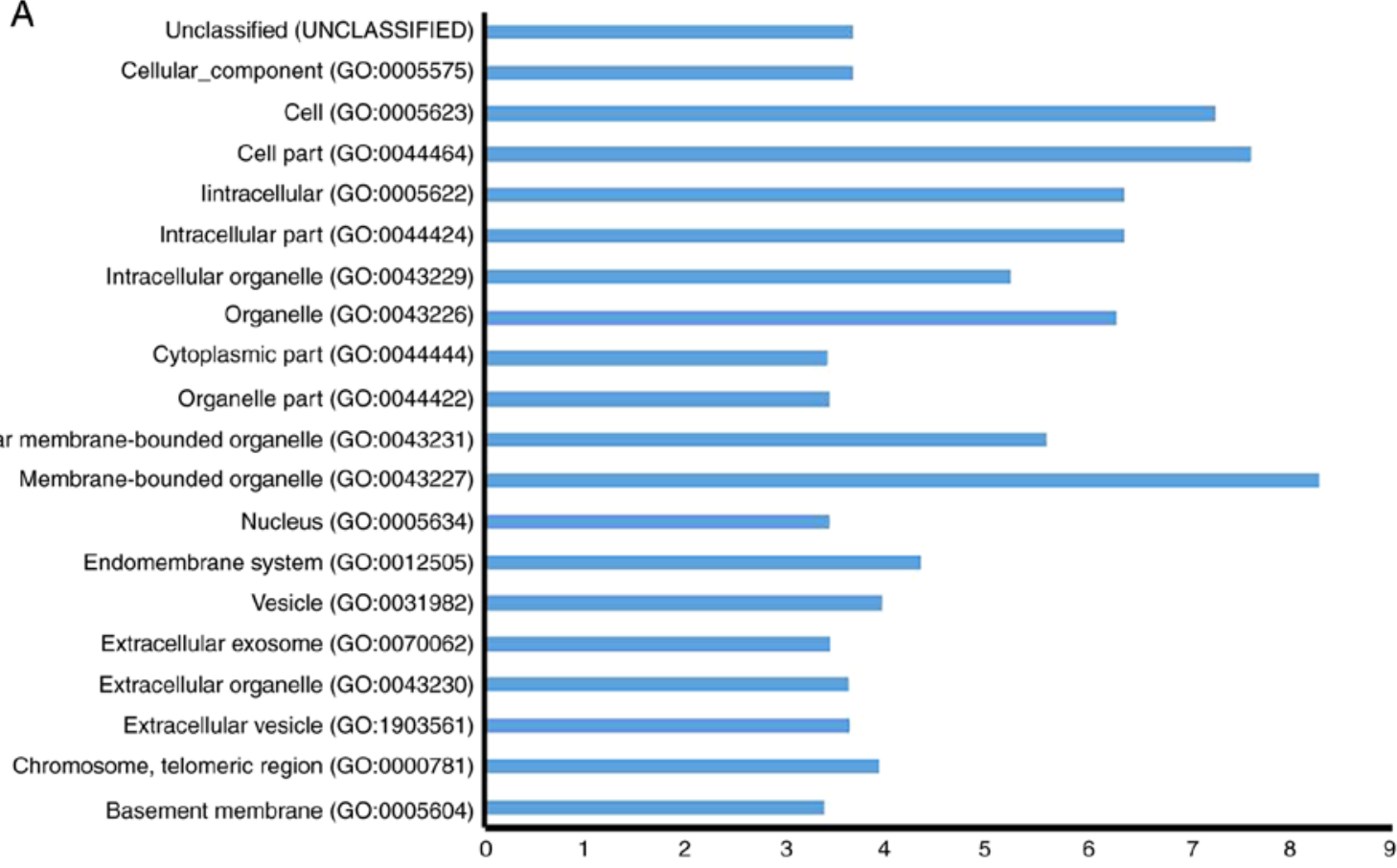

B Intracellular membrane-bounded organelle (GO:0043231) Membrane-bounded organelle (GO:0043227)

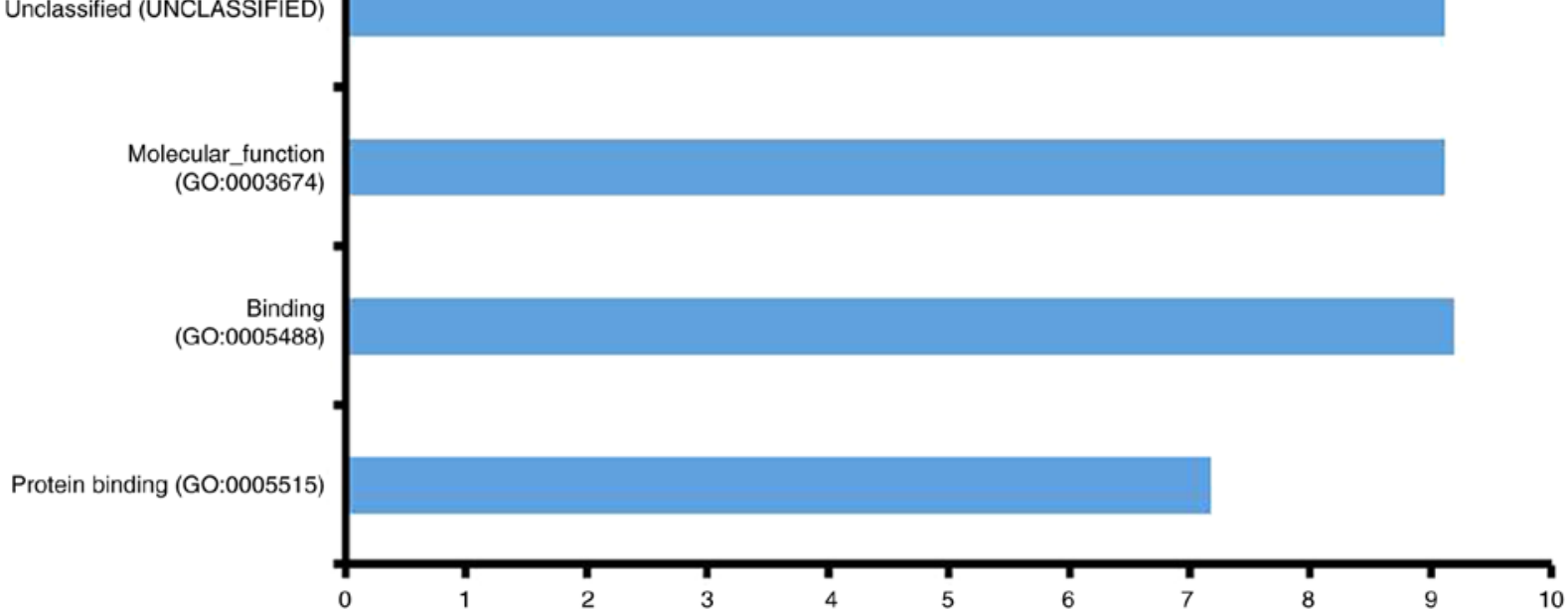

C

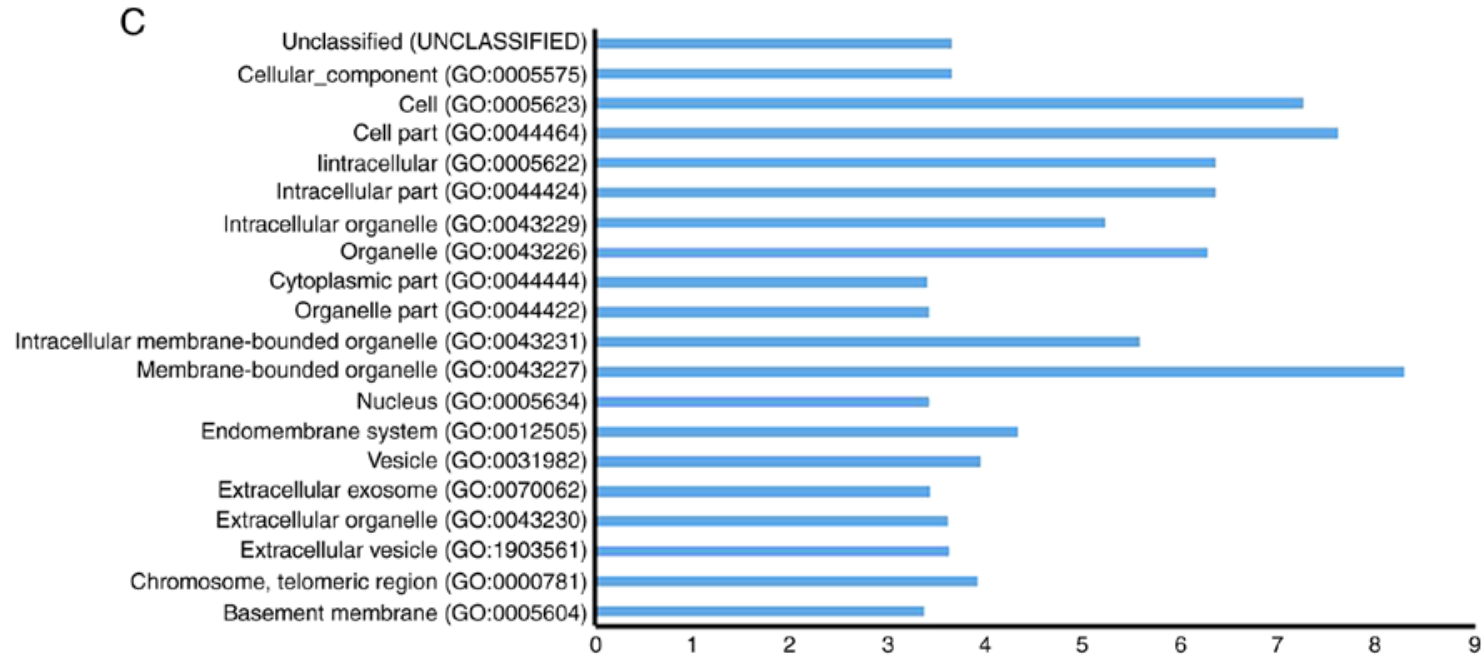

Figure 1. The function of genes collected from Gene Expression Omnibus (GEO) database were determined using geometric means of semantic similarities in biological processes (BPs), molecular functions (MFs), and cellular components (CCs), as measured using the GOSemSim package by considering the Gene Ontology (GO) topological structure in a more precise and unbiased manner. (A) The peak of biological processes (BPs). (B) The peak of molecular functions (MFs). (C) The peak of cellular components (CCs). 

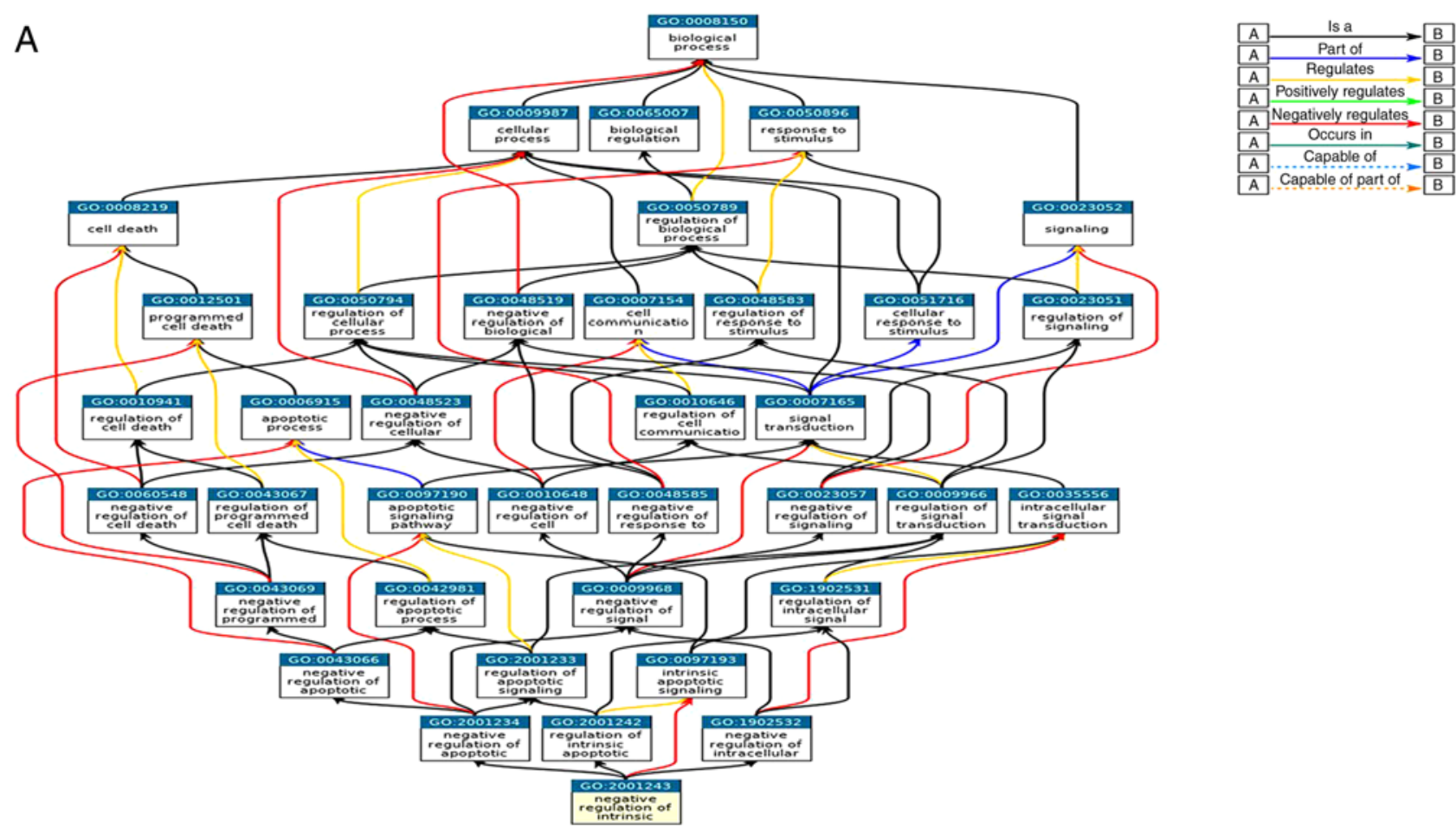

B
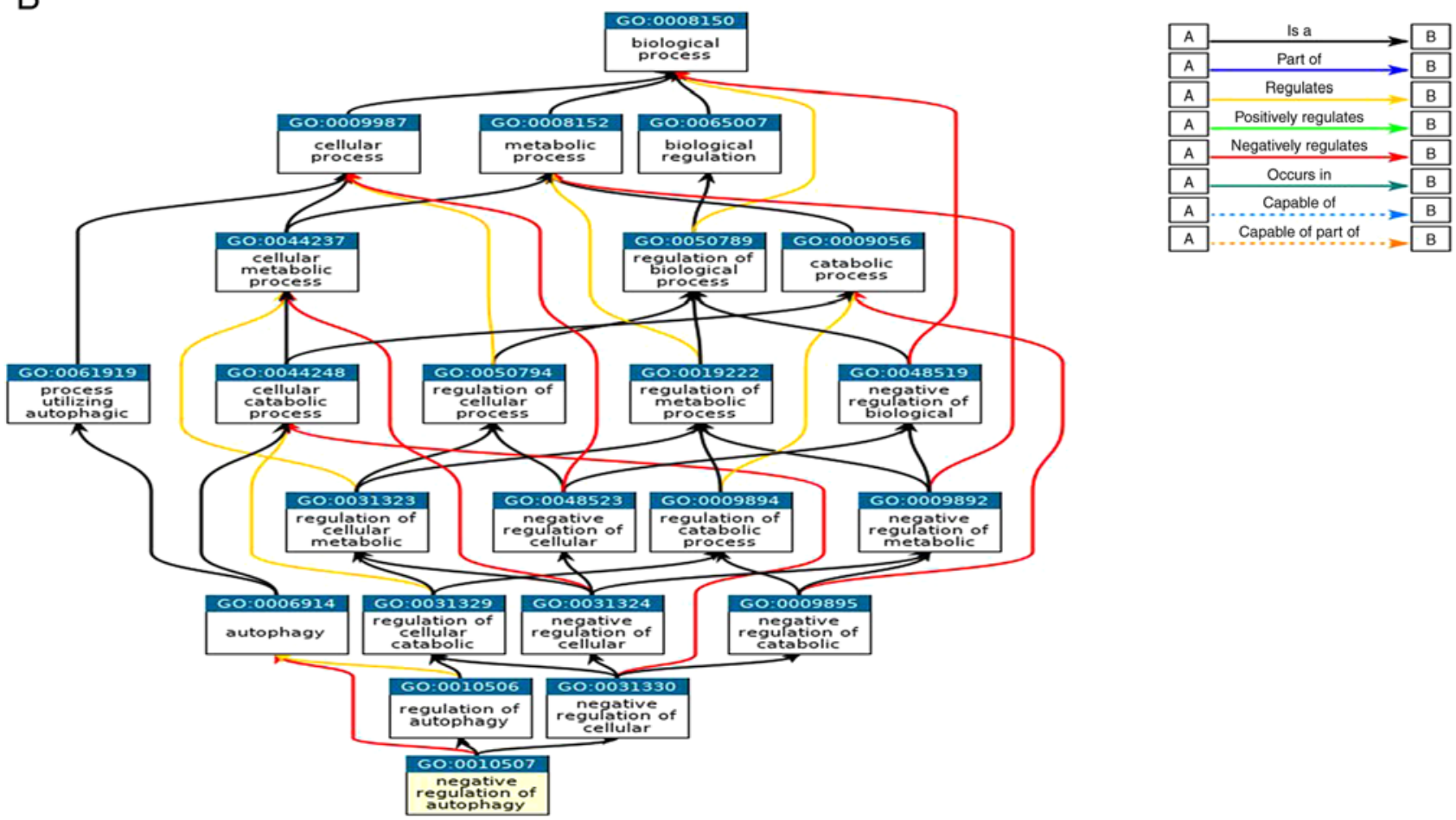

Figure 2. Gene Ontology (GO) items related to the regulation of apoptosis and autophagy. (A) Relationship of GO items in the regulation of apoptosis. (B) Relationship of GO items in the regulation of autophagy.

pathway, the P53 pathway, and others. During analysis, SIRT1 was also found to be related to the AMPK pathway and autophagy pathway, consistent with findings from our previous study (23).

A total of 317 regulator genes, together with lysine-specific demethylase 4A (KDM4A), a regulation protein for the H3K9me3 network containing 220 nodes and 202 edges, were obtained from the STRING online database and Cytoscape software. The PPI network showed detailed protein interactions; however, the PPI enrichment P-value was above 0.05 , suggesting that the network did not have significantly more interactions than expected. Nevertheless, the modulation enzyme lysine-specific demethylase 4A (KDM4A) for $\mathrm{H} 3 \mathrm{~K} 9 \mathrm{me} 3$ could have an interaction with other proteins in the network by HISTIH4C (Histone cluster 1, H4c, a modulation enzyme for histone $\mathrm{H} 4$ ). There were also many protein interactions that could help identify the mechanism of H3K9me3 modulation (Fig. 4). 


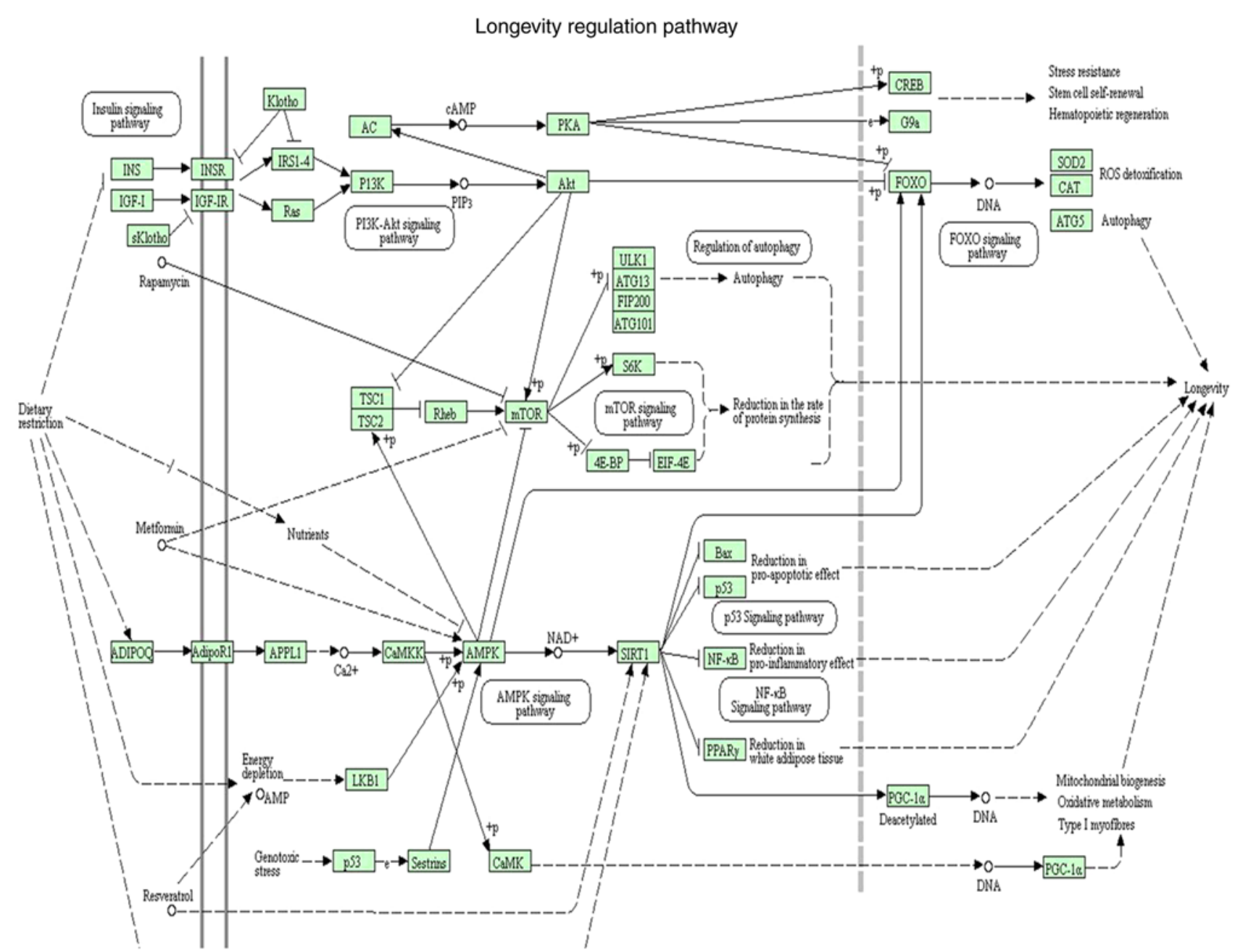

Figure 3. Related pathways were predicted using KEGG Orthology-Based Annotation System (KOBAS) 3.0 online. This pathway was called the longevity pathway. The pathway contained regular pathways related to the survival of leukaemia cells, including the PI3K/AKT pathway, the AMPK pathway, the FOXO pathway, the P53 pathway, mTOR pathway and NF- $\mathrm{KB}$ pathway. During analysis, SIRT1 was found to be related to the AMPK pathway or the autophagy pathway, consistent with our previous studies. PI3K, phosphatidylinositol 3-kinase; AKT, serine/threonine kinase; AMPK, protein kinase AMP-activated catalytic subunit $\alpha 1$; FOXO, forkhead box; mTOR, mechanistic target of rapamycin kinase; SIRT1, sirtuin 1.

Distribution of peaks for gene function and the analysis of $G O$ using KEGG network in AML cells treated with chidamide. The ChIP-seq of H3K9me3 was performed in AML cells in which levels of SIRT1 were inhibited by chidamide; this was conducted to identify genes that may be involved in the mechanisms of cell death. The peaks almost clustered in the gene and intergenic areas (nearly 99\%); others clustered in areas such as CDS and D10K20K (Fig. 5A).

$\mathrm{GO}$ analysis showed that the peak of processes that had significant changes for AML cells treated by chidamide were BPs and MFs. In the BPs, the most significant peaks involved metabolic processes such as the macromolecule metabolic process and cellular macromolecule metabolic processes. In the MFs, the most significant peaks involved metal ion binding or cation binding. KEGG analysis also revealed that the richest peak involved metabolic processes (Fig. 5B).

Network analysis of GO enrichment in AML cells treated by chidamide were in BPs. Units responsive to stress in directed acyclic graph (DAG) had the most significant changes, especially for the DNA repair and cells responsive to stress. In the CCs, the ribosome unit showed the most significant change; other units also showed significant changes, including cytoplasm and intracellular ribonucleoprotein. In the MFs, phosphotransferase, nucleotide kinase, and adenylate kinase showed the most significant changes.

Our previous research (13) suggested that the level of H3K9me3 could be affected by a low dose of chidamide, thus we aimed to ascertain in the present study whether the network of GO enrichment in AML cells similar to AML patients could be affected by chidamide. This result may be evidence for further research concerning the use of chidamide in AML patients. An interesting result was that the network analysis of GO enrichment in AML cells treated with vhidamide were similar in AML patients. In GO0050789, GO0050794, GO0050896, and GO0080090, all of which are related to BPs, GO enrichment showed significant changes in AML patients compared with control groups; the same also appeared in AML cells treated with chidamide (Fig. 5E), which was different from THP-1 cells without chidamide treatment (Fig. 5C). In the CCs, GO enrichment such as GO005623, GO005622, and GO0044444 showed significant changes in AML patients as well as in the AML cells treated with chidamide. However, 


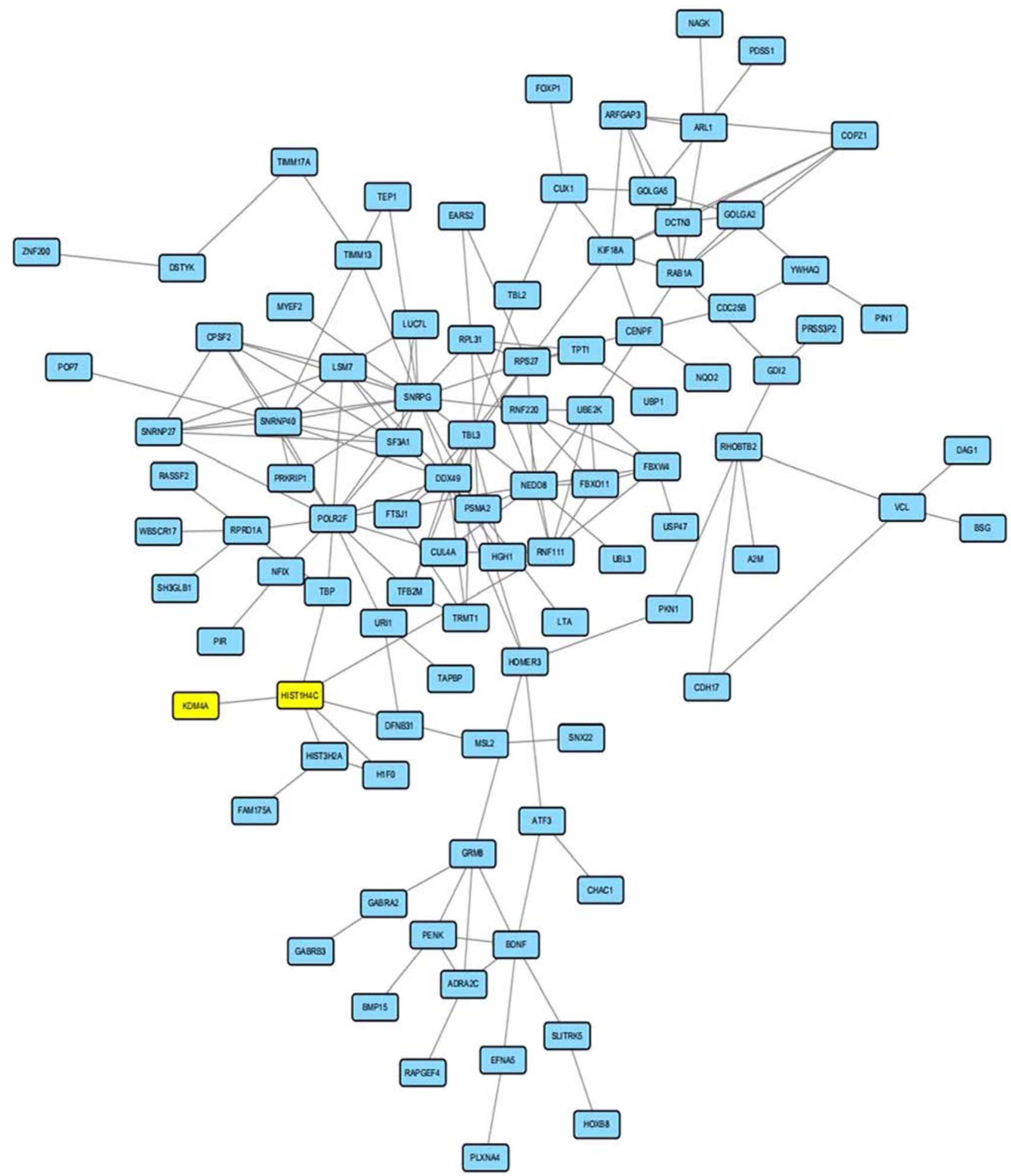

Figure 4. Interaction analysis for regulator genes together with KDM4A demonstrated by software Cytoscape 3.5.1. The protein-protein interaction (PPI) network of regulator genes together with KDM4A showing detailed protein interactions; however, the PPI enrichment P-value was $>0.05$, suggesting that the network did not have significantly more interactions than expected. KDM4A, lysine-specific demethylase 4A; HISTIH4C, histone cluster 1, H4c.

in MFs, only GO005488 showed significant changes in AML patients and in AML cells treated with chidamide (Fig. 5F), which was different from THP-1 cells without chidamide treatment (Fig. 5D).

Interactions between SIRT1 and H3K9me3 regulatory proteins that showed significant changes in AML patients.
The network of analysis for AML cells treated with chidamide showed several results of GO enrichment that were the same as the results of GO enrichment obtained from the patients in GSE20452. Our previous study showed that SIRT1 (located on chromosome 10) was in the domain of H3K9me3 on chromosome 10 that was downregulated by chidamide, suggesting there may be interaction between SIRT1 and the regulation of 


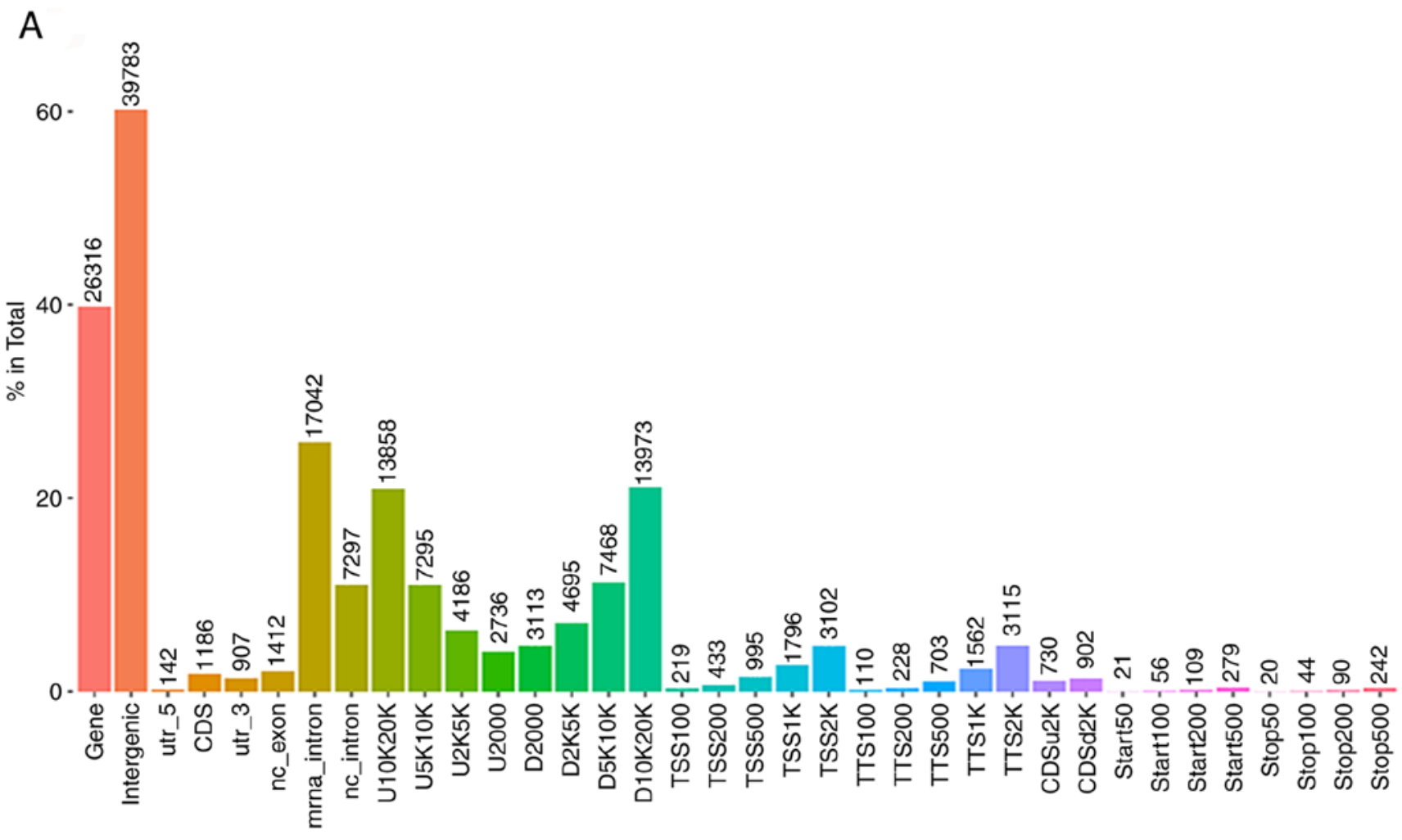

B

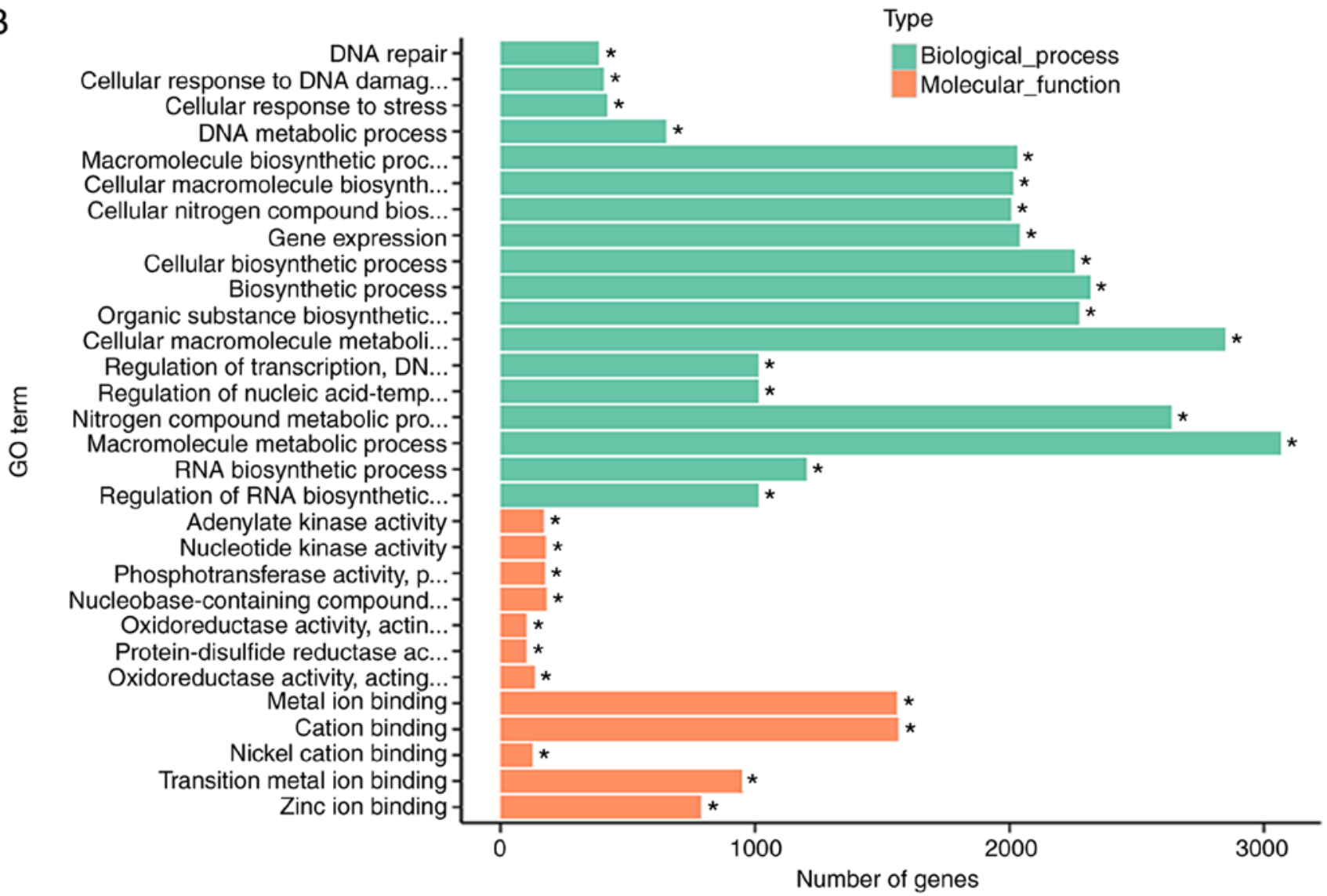

Figure 5. Function of genes collected from acute myeloid leukaemia (AML) cells tested in ChIP-seq were further calculated using the geometric mean of semantic similarities in biological processes (BPs) and molecular functions (MFs), as determined using the GOSemSim package by considering the Gene Ontology (GO) topological structure in a more precise and unbiased manner. (A) The distribution of the peak for gene function. The ratio of the peak almost clustered in the gene and intergenic areas (nearly 99\%); others clustered in the areas such as CDS and D10K20K. (B) The peak of biological processes (BPs) and the peak of molecular functions (MFs). 
C
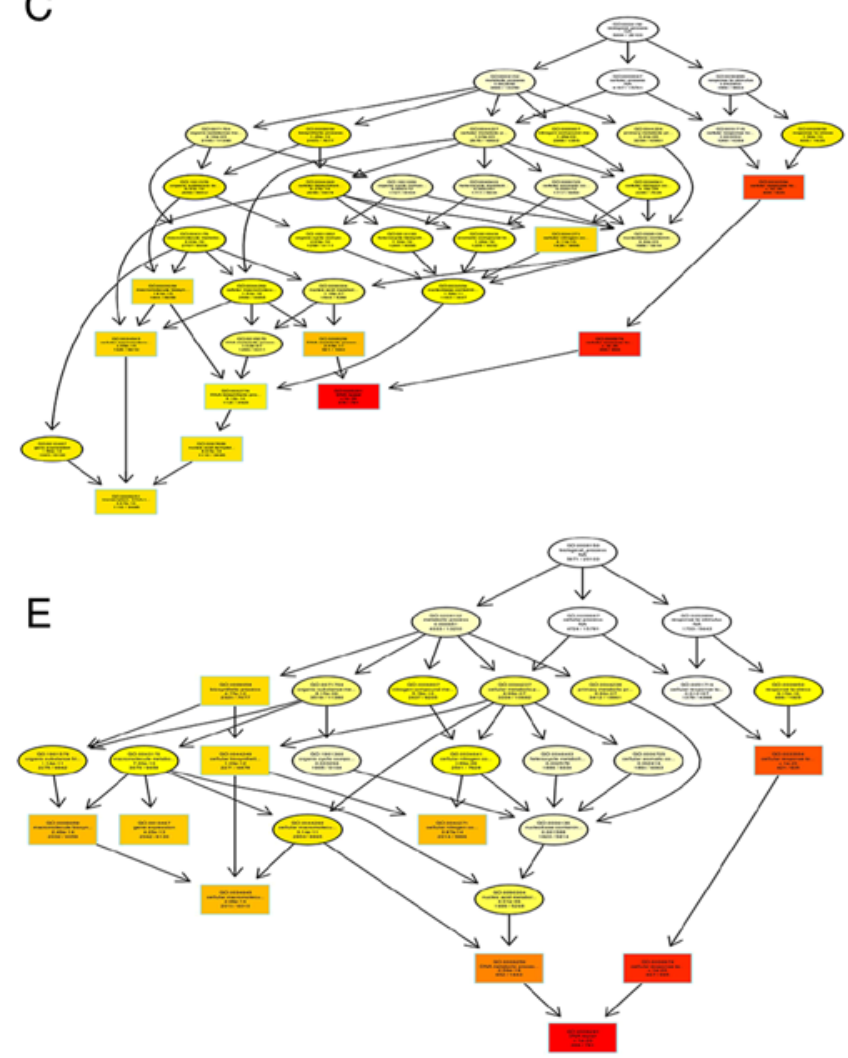
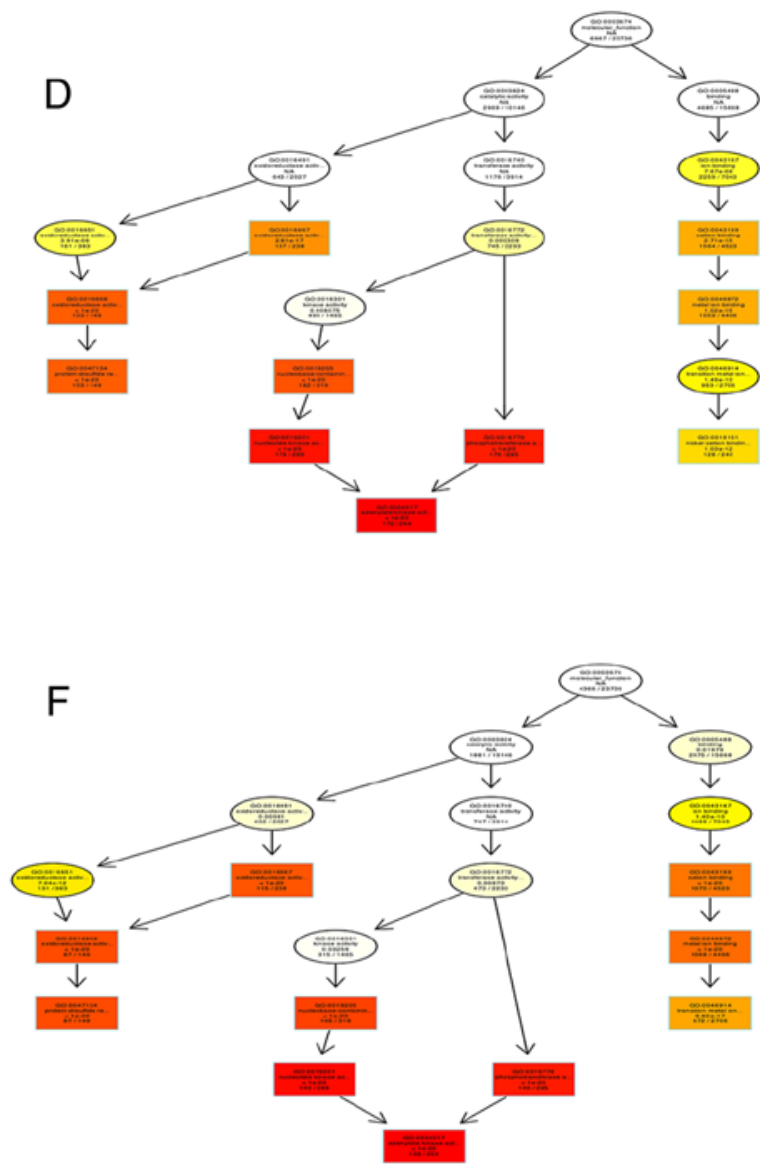

Figure 5. Continued. (C) Relationship of GO items in BPs of THP-1 cells without chidamide treatment. (D) Relationship of GO items in MFs of THP-1 cells without chidamide treatment. (E) Relationship of GO items in the BPs of THP-1 cells treated with chidamide. (F) Relationship of GO items in MFs of THP-1 cells treated with chidamide. In this part, some GO items in BPs or MFs were the same as GO items in AML patients. CDS, CDP-diacylglycerol synthase.

H3K9me3 (17). A total of 317 regulator genes together with SIRT1, containing 219 nodes and 209 edges, were obtained from the STRING online database and Cytoscape software. The PPI network showed detailed protein interactions; however, the PPI enrichment P-value was above 0.05, suggesting that the network did not have significantly more interactions than expected. Nevertheless, SIRT1 had a direct interaction with several proteins in the network compared with KDM4A; however, there were also many other protein interactions that were identified so as to uncover the relationship between SIRT1 and regulatory genes acting on H3K9me3 (Fig. 6A).

Interactions between SIRT1 and KDM4A. Our previous study (13) showed that the location of SIRT1 on chromosome 10 was in the domain of $\mathrm{H} 3 \mathrm{~K} 9 \mathrm{me}$, suggesting there also may exist interactions between SIRT1 and KDM4A. The gene SIRT1, together with KDM4A, contained eight nodes and seven edges, obtained from STRING online database and Cytoscape software. The PPI network showed the detailed protein interactions, and the PPI enrichment P-value was below 0.05 , suggesting that the network had significantly more interactions than expected. Nevertheless, there were no direct interactions between SIRT1 and KDM4A. The protein M-phase phosphoprotein 8 (MPHOSPH8) may be the bridge for SIRT1 and KDM4A, and interactions between MPHOSPH8 and KDM4A need to be demonstrated (Fig. 6B).

\section{Discussion}

Histone $\mathrm{H} 3$ lysine 9 trimethylation (H3K9me3) has a role not only in malignancies but also in normal cellular development. It acts as a repressor of lineage-inappropriate genes and it maintains early cell integrity and genomic stability. In the early 2000s (24), a number of groups provided evidence of its important interactions with evolutionarily conserved amino terminal chromodomain of heterochromatin protein 1 (HP1), a hallmark of heterochromatin, thereby recruiting it to specific chromatin loci. To date, roles for $\mathrm{H} 3 \mathrm{~K} 9 \mathrm{me} 3$ have been revealed in regulating apoptosis $(25,26)$, autophagy $(27)$, development $(28,29)$, DNA repair (30-33), splicing (34-38), self-renewal $(39,40)$, transcriptional elongation (36), viral latency (41-43), imprinting (44), aging (45), and cell identity (46). In acute myeloid leukaemia (AML), alterations in H3K9 methylation at promoter regions were found to be associated with inactivation of tumour-suppressor genes and blockade of differentiation and deregulated proliferation $(47,48)$. Given the reversible nature of $\mathrm{H} 3 \mathrm{~K} 9$ trimethylation, this represents an attractive therapeutic target in AML.

Correct identification of the signalling pathways in AML is the foundation of the discovery of therapeutic targets for $\mathrm{H} 3 \mathrm{~K} 9 \mathrm{me}$. In the present study, data from AML patients from the GEO dataset were analysed using GEOR2. Compared with the control group (CD34+ ${ }^{+}$white cells), there were several 
A

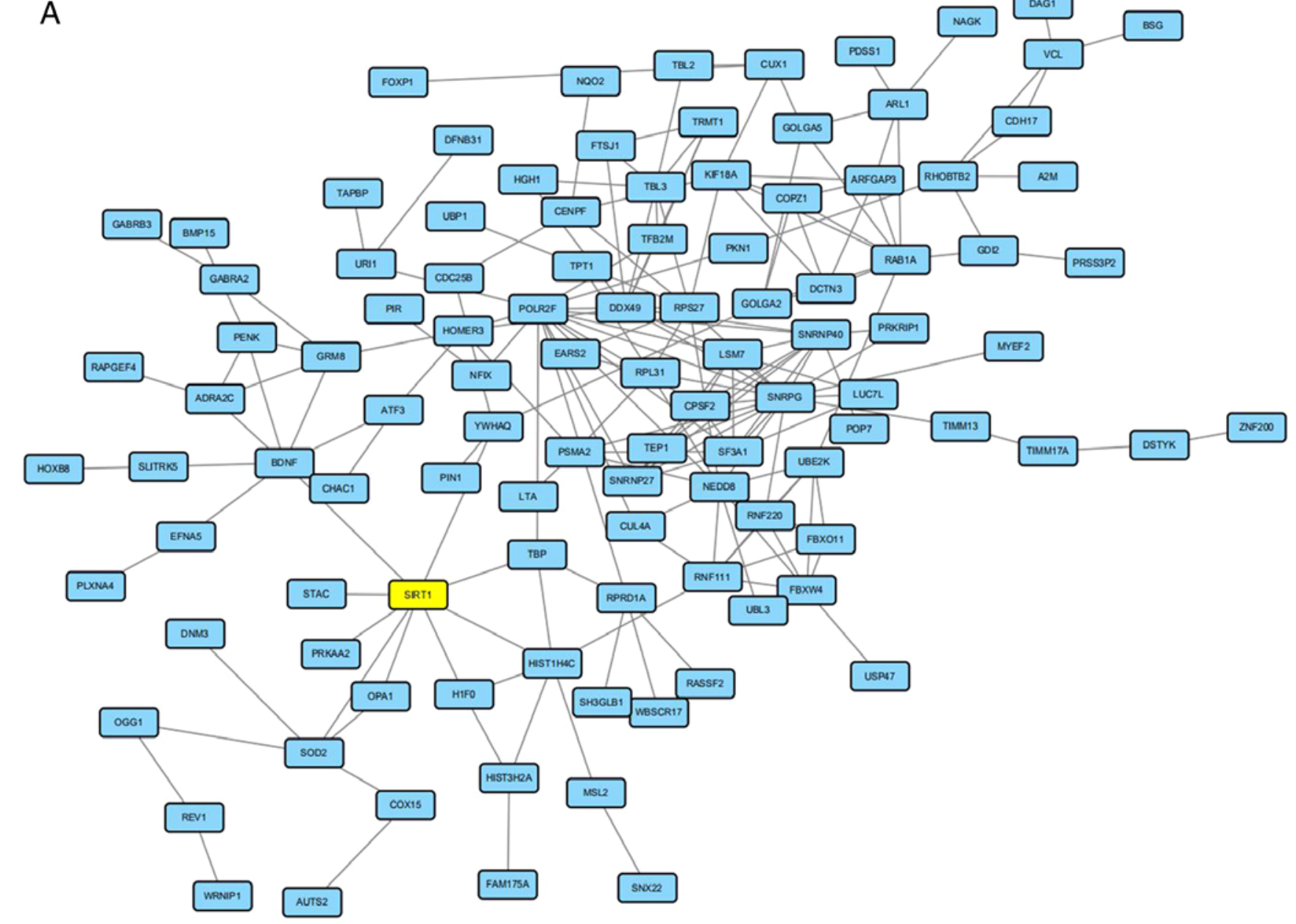

B
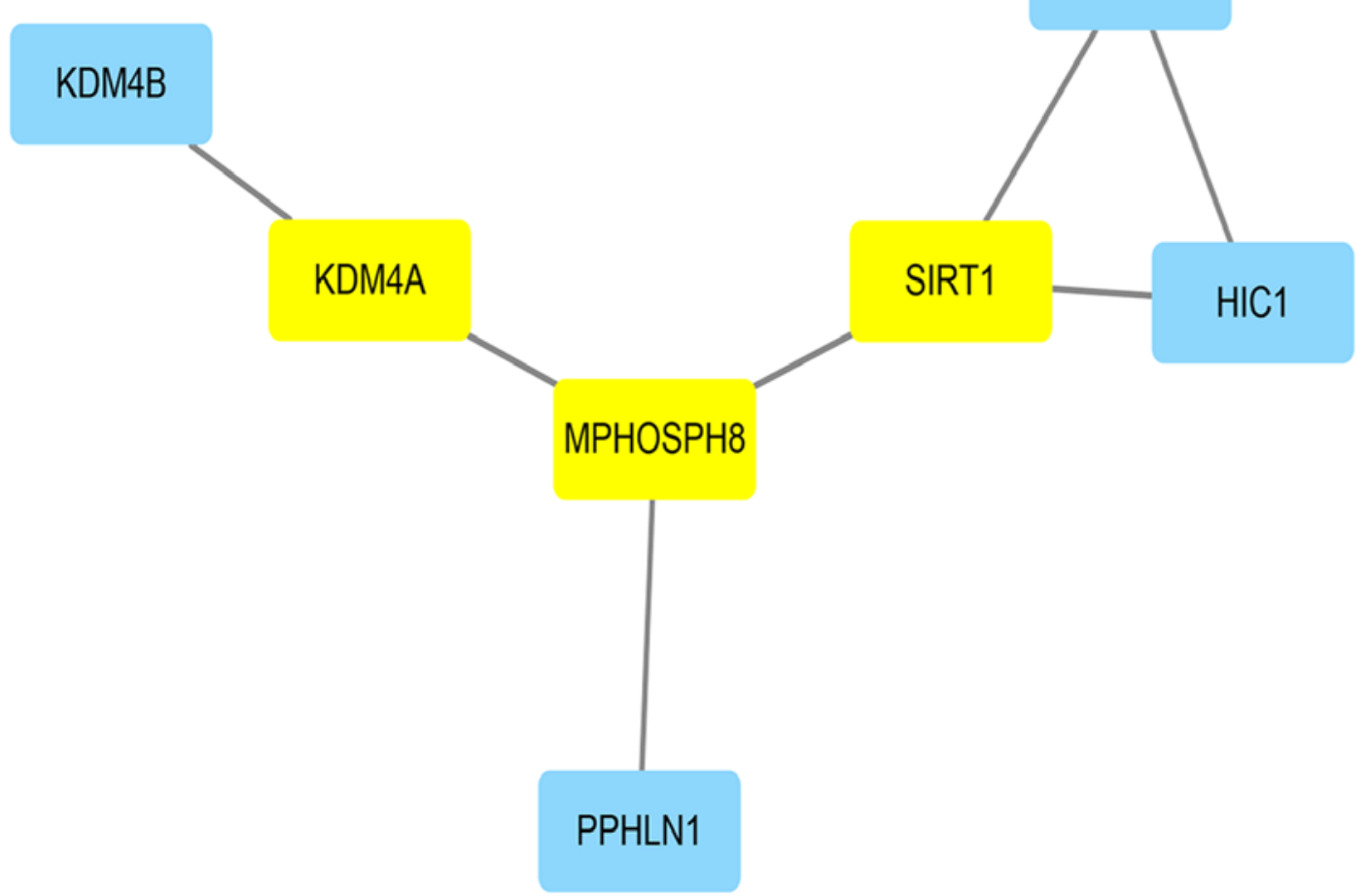

Figure 6. Interaction analysis for regulator genes together with SIRT1 and the interaction analysis for SIRT1 together with KDM4A as demonstrated by software Cytoscape 3.5.1. (A) The PPI network of regulator genes together with SIRT1 showing detailed protein interactions. The PPI enrichment P-value was $>0.05$, suggesting that the network did not have significantly more interactions than expected. (B) The interaction analysis for SIRT1 together with KDM4A showing that SIRT1 may have an indirect interaction with KDM4A, suggesting that the network had significantly more interactions than expected. KDM4A, lysine-specific demethylase 4A; SIRT1, sirtuin 1. 
genes related to changes in $\mathrm{H} 3 \mathrm{~K} 9 \mathrm{me} 3$ that were significantly differentially upregulated or downregulated. These genes were related to biological processes (BPs), molecular functions (MFs), and cellular components (CCs). Some genes were related to the development of AML and some took part in drug resistance in AML. These changes showed that the change in histone methylation also may be an important factor for the development of AML or drug resistance. In the present study, various changes in THP-1 cells treated with chidamide were the same as that in AML patients. The network analysis of $\mathrm{GO}$ enrichment showed that alterations in $\mathrm{H} 3 \mathrm{k} 9 \mathrm{me} 3$ may cause changes in cells in regards to apoptosis, autophag. These databases became a potential foundation for our subsequent analysis of chidamide on AML cells in vitro. THP-1 is a cell line that is resistant to cytarabine, and chidamide could enhance the cytotoxicity of cytarabine in THP-1 cells by modulating $\mathrm{H} 3 \mathrm{~K} 9 \mathrm{me} 3$ (13). $\mathrm{H} 3 \mathrm{~K} 9 \mathrm{me} 3$ was also reported to be related to poor patient prognosis, thus it was thought that $\mathrm{H} 3 \mathrm{~K} 9 \mathrm{me} 3$ may be related to drug-resistance which is a main factor for poor prognosis (12). There are no studies concerning similar changes in gene expression in other AML cell lines that have been carried out. Changes in the THP-1 cell line in this research may give us a direction for further research in regards to other drug-resistant AML cell lines. The functions of gene expression changes in other drug-resistant AML cells will be carried out in next stage in future research.

The interaction analysis for related proteins of $\mathrm{H} 3 \mathrm{~K} 9 \mathrm{me} 3$ showed only a few proteins with interactions that had been previously demonstrated in other studies (49-51). The modulation protein lysine-specific demethylase 4A (KDM4A) for $\mathrm{H} 3 \mathrm{~K} 9 \mathrm{me} 3$ in this analysis was found only with an interaction with HISTIH4C that was a modulation enzyme for another histone (H4). These findings suggest that there are many interactions between KDM4A and other proteins that warrant further investigation. Meanwhile further research concerning the function of KDM4A on AML cells will be carried out in subsequent research.

A recent review highlighted the emerging theme that histone modifications can influence one another, such that one modification recruits or activates chromatin-modifying complexes to generate additional histone modifications (12). Our previous study also showed that the drug chidamide (a histone deacetylation inhibitor (HDACi) developed in China) was the first oral subtype-selective HDACi in the world that could enhance the cytotoxicity of drugs in AML cells (17). One of the potential mechanisms might be related to an effect on H3K9me3. The data suggest that there may be the same changes in vitro as those in patients. The ChIP-seq test for THP-1 cells treated with chidamide showed that significant peaks of GO analysis were BPs and MFs, which lack the course of CCs compared with the results in patients. However, the network analysis of GO enrichment in vitro found that some changes were related to apoptosis and autophagy. As previously suspected, some forecast changes in vitro were the same as the forecast changes in patients, including GO0050789, GO0050794, GO0050896, and GO0080090, related to the BPs or the GO enrichment such as GO005623, GO005622, and GO0044444 in the CCS. These results may be a theoretical basis for further usage of chidamide in AML patients. We believe that these results may suggest target treatments involving $\mathrm{H} 3 \mathrm{~K} 9 \mathrm{me} 3$.
Changes in H3K9me3 in patients or in vitro may cause autophagy. Our previous study regarding the potential mechanisms of action of chidamide in enhancing the cytotoxicity of drugs in AML cells suggested that chidamide inhibits autophagy by inhibiting sirtuin 1 (SIRT1), a histone deacetylation enzyme (17). SIRT1 may also have an interaction with changes in $\mathrm{H} 3 \mathrm{k} 9 \mathrm{me} 3$ or the modulation enzyme KDM4A. The pathway of KEGG analysis of H3k9me3 in patients showed several pathways related to the survival of leukaemia cells. These included some related to the survival of leukaemia cells, including the PI3K/AKT pathway, the AMPK pathway, the FOXO pathway, the P53 pathway, and others. SIRT1 had an effect on the FOXO pathway that was downstream of the PI3K/AKT pathway, related to drug resistance. This result may be evidence to support the mechanism of chidamide in reversing drug resistance in AML cells via the SIRTI gene. This research is currently being conducted by our research group. In the STRING database analysis, the interaction of SIRT1 with proteins related to a change in $\mathrm{H} 3 \mathrm{~K} 9 \mathrm{me} 3$ were more evident even more than KDM4A; however, the network did not have significantly more interactions than expected, suggesting that further research needs to be conducted. A compelling result for the interaction of SIRT1 with KDM4A may be a relationship between SIRT1 and KDM4A, although there was not a direct interaction, and there may be involvement of a bridge called the MPHOSPH8 gene. Research for further verification of the relationship between KDM4A and MPHOSPH8 or SIRT1 must be carried out. This result also suggests a link between histone deacetylation and methylation, as reported in other studies about histone modifications influencing one another (52-55).

In conclusion, bioinformatics analysis of $\mathrm{H} 3 \mathrm{k} 9 \mathrm{me} 3$ in patients and in AML cells in vitro showed that $\mathrm{H} 3 \mathrm{~K} 9 \mathrm{me} 3$ may be a target for the treatment for AML; it also suggested that chidamide may be a target drug for AML patients. Finally, our data suggest several directions for the further study of drug resistance in AML.

\section{Acknowledgements}

\section{Not applicable.}

\section{Funding}

This research study was supported by Zhejiang Natural Science Foundation Program (LY19H080002), and the Breeding Program of the Second Affiliated Hospital and Yumiao Children's Hospital of Wenzhou Medical University (Wenzhou, Zhejiang, China).

\section{Availability of data and materials}

We declared that materials described in the manuscript, including all relevant raw data, will be freely available to any scientist wishing to use them for non-commercial purposes, without breaching participant confidentiality.

\section{Authors' contributions}

$\mathrm{AD}, \mathrm{WY}$ and WG performed the analysis of the genes for the GEO database peak analysis, as well as analysis of protein interactions. 
$\mathrm{HH}, \mathrm{ZH}$ and XZ performed the ChIP-seq analysis. RY conducted the statistical analysis. WG wrote the paper. All authors read and approved the manuscript and agree to be accountable for all aspects of the research in ensuring that the accuracy or integrity of any part of the work are appropriately investigated and resolved.

\section{Ethics approval and consent to participate}

This article does not contain any studies with human participants or animals performed by any of the authors.

\section{Patient consent for publication}

Not applicable.

\section{Competing interests}

The authors declare that they have no competing interests.

\section{References}

1. Chen W, Zheng R, Baade PD, Zhang S, Zeng H, Bray F, Jemal A Yu XQ and He J: Cancer statistics in China, 2015. CA Cancer J Clin 66: 115-132, 2016

2. Goyama S and Kitamura T: Epigenetics in normal and malignant hematopoiesis: An overview and update 2017. Cancer Sci 108: 553-562, 2017

3. Cao R, Wang L, Wang H, Xia L, Erdjument-Bromage H, Tempst P, Jones RS and Zhang Y: Role of histone H3 lysine 27 methylation in Polycomb-group silencing. Science 298: 1039-1043, 2002.

4. Morin RD, Johnson NA, Severson TM, Mungall AJ, An J, Goya R, Paul JE, Boyle M, Woolcock BW, Kuchenbauer F, et al: Somatic mutations altering EZH2 (Tyr641) in follicular and diffuse large B-cell lymphomas of germinal-center origin. Nat Genet 42: 181-185, 2010.

5. Abd Al Kader L, Oka T, Takata K, Sun X, Sato H, Murakami I, Toji T, Manabe A, Kimura $\mathrm{H}$ and Yoshino T: In aggressive variants of non-Hodgkin lymphomas, Ezh2 is strongly expressed and polycomb repressive complex PRC1.4 dominates over PRC1.2. Virchows Arch 463: 697-711, 2013.

6. Asangani IA, Ateeq B, Cao Q, Dodson L, Pandhi M, Kunju LP, Mehra R, Lonigro RJ, Siddiqui J, Palanisamy N, et al Characterization of the EZH2-MMSET histone methyltransferase regulatory axis in cancer. Mol Cell 49: 80-93, 2013.

7. Ernst T, Chase AJ, Score J, Hidalgo-Curtis CE, Bryant C, Jones AV, Waghorn K, Zoi K, Ross FM, Reiter A, et al Inactivating mutations of the histone methyltransferase gene EZH2 in myeloid disorders. Nat Genet 42: 722-726, 2010.

8. Nikoloski G, Langemeijer SM, Kuiper RP, Knops R, Massop M, Tönnissen ER, van der Heijden A, Scheele TN, Vandenberghe P, de Witte T, et al: Somatic mutations of the histone methyltransferase gene EZH2 in myelodysplastic syndromes. Nat Genet 42: 665-667, 2010.

9. Ntziachristos P, Tsirigos A, Van Vlierberghe P, Nedjic J, Trimarchi T, Flaherty MS, Ferres-Marco D, da Ros V, Tang Z, Siegle J, et al: Genetic inactivation of the polycomb repressive complex 2 in $T$ cell acute lymphoblastic leukemia. Nat Med 18: 298-301, 2012.

10. Daigle SR, Olhava EJ, Therkelsen CA, Basavapathruni A, Jin L, Boriack-Sjodin PA, Allain CJ, Klaus CR, Raimondi A, Scott MP, et al: Potent inhibition of DOT1L as treatment of MLL-fusion leukemia. Blood 122: 1017-1025, 2013.

11. Muntean AG and Hess JL: The pathogenesis of mixed-lineage leukemia. Annu Rev Pathol 7: 283-301, 2012.

12. Müller-Tidow C, Klein HU, Hascher A, Isken F, Tickenbrock L, Thoennissen N, Agrawal-Singh S, Tschanter P, Disselhoff C, Wang Y, et al; Study Alliance Leukemia: Profiling of histone H3 lysine 9 trimethylation levels predicts transcription factor activity and survival in acute myeloid leukemia. Blood 116: 3564-3571, 2010

13. Huang H, Wenbing Y, Dong A, He Z, Yao R and Guo W: Chidamide enhances the cytotoxicity of cytarabine and sorafenib in acute myeloid leukemia cells by modulating H3K9me3 and autophagy levels. Front Oncol 9: 1276, 2019.
14. Xie C, Mao X, Huang J, Ding Y, Wu J, Dong S, Kong L, Gao G, Li CY and Wei L: KOBAS 3.0: A web server for annotation and identification of enriched pathways and diseases. Nucleic Acids Res 39: W316-W322, 2011.

15. Kanehisa M and Goto S: KEGG: Kyoto encyclopedia of genes and genomes. Nucleic Acids Res 28: 27-30, 2000.

16. Kanehisa M, Sato Y, Furumichi M, Morishima K and Tanabe M: New approach for understanding genome variations in KEGG. Nucleic Acids Res 47: D590-D595, 2019.

17. Kanehisa M: Toward understanding the origin and evolution of cellular organisms. Protein Sci 28: 1947-1951, 2019.

18. Szklarczyk D, Franceschini A, Wyder S, Forslund K, Heller D, Huerta-Cepas J, Simonovic M, Roth A, Santos A, Tsafou KP, et al: STRING v10: Protein-protein interaction networks, integrated over the tree of life. Nucleic Acids Res 43: D447-D452, 2015

19. Shannon P, Markiel A, Ozier O, Baliga NS, Wang JT, Ramage D, Amin N, Schwikowski B and Ideker T: Cytoscape: A software environment for integrated models of biomolecular interaction networks. Genome Res 13: 2498-2504, 2003.

20. Li X, Yan X, Guo W, Huang X, Huang J, Yu M, Ma Z, Xu Y, Huang S, Li C, et al: Chidamide in FLT3-ITD positive acute myeloid leukemia and the synergistic effect in combination with cytarabine. Biomed Pharmacother 90: 699-704, 2017.

21. Kleine-Kohlbrecher D, Christensen J, Vandamme J, Abarrategui I Bak M, Tommerup N, Shi X, Gozani O, Rappsilber J, Salcini AE and Helin K: A functional link between the histone demethylase PHF8 and the transcription factor ZNF711 in X-linked mental retardation. Mol Cell 38: 165-178, 2010.

22. Yu G, Li F, Qin Y, Bo X, Wu Y and Wang S: GOSemSim: an R package for measuring semantic similarity among GO terms and gene products. Bioinformatics 26: 976-978, 2010.

23. Guo W, Jin J, Pan J, Yao R, Li X, Huang X, Ma Z, Huang S, Yan X, Jin J and Dong A: The change of nuclear LC3 distribution in acute myeloid leukemia cells. Exp Cell Res 369: 69-79, 2018.

24. Mandrioli M and Borsatti F: Analysis of heterochromatic epigenetic markers in the holocentric chromosomes of the aphid Acyrthosiphon pisum. Chromosome Res 15: 1015-1022, 2007.

25. Olcina MM, Leszczynska KB, Senra JM, Isa NF, Harada $H$ and Hammond EM: H3K9me3 facilitates hypoxia-induced p53-dependent apoptosis through repression of APAK. Oncogene 35: 793-799, 2016.

26. Lu C, Yang D, Sabbatini ME, Colby AH, Grinstaff MW, Oberlies NH, Pearce C and Liu K: Contrasting roles of H3K4me3 and $\mathrm{H} 3 \mathrm{~K} 9 \mathrm{me} 3$ in regulation of apoptosis and gemcitabine resistance in human pancreatic cancer cells. BMC Cancer 18: 149, 2018.

27. Biga PR, Latimer MN, Froehlich JM, Gabillard JC and Seiliez I: Distribution of $\mathrm{H} 3 \mathrm{~K} 27 \mathrm{me} 3, \mathrm{H} 3 \mathrm{~K} 9 \mathrm{me} 3$, and $\mathrm{H} 3 \mathrm{~K} 4 \mathrm{me} 3$ along autophagy-related genes highly expressed in starved zebrafish myotubes. Biol Open 6: 1720-1725, 2017.

28. Fujiwara K, Fujita Y, Kasai A, Onaka Y, Hashimoto H, Okada H and Yamashita T: Deletion of JMJD2B in neurons leads to defective spine maturation, hyperactive behavior and memory deficits in mouse. Transl Psychiatry 6: e766, 2016.

29. Magaraki A, van der Heijden G, Sleddens-Linkels E, Magarakis L, van Cappellen WA, Peters AHFM, Gribnau J, Baarends WM and Eijpe M: Silencing markers are retained on pericentric heterochromatin during murine primordial germ cell development. Epigenetics Chromatin 10: 11, 2017.

30. Sun Y, Jiang X, Xu Y, Ayrapetov MK, Moreau LA, Whetstine JR and Price BD: Histone H3 methylation links DNA damage detection to activation of the tumour suppressor Tip60. Nat Cell Biol 11: 1376-1382, 2009.

31. Ayrapetov MK, Gursoy-Yuzugullu O, Xu C, Xu Y and Price BD: DNA double-strand breaks promote methylation of histone $\mathrm{H} 3$ on lysine 9 and transient formation of repressive chromatin. Proc Natl Acad Sci USA 111: 9169-9174, 2014.

32. Khoury-Haddad H, Nadar-Ponniah PT, Awwad S and Ayoub N: The emerging role of lysine demethylases in DNA damage response: Dissecting the recruitment mode of KDM4D/JMJD2D to DNA damage sites. Cell Cycle 14: 950-958, 2015.

33. Wu R, Wang Z, Zhang H, Gan $\mathrm{H}$ and Zhang Z: H3K9me3 demethylase Kdm4d facilitates the formation of pre-initiative complex and regulates DNA replication. Nucleic Acids Res 45: 169-180, 2017.

34. Hausmann M, Wagner E, Lee JH, Schrock G, Schaufler W, Krufczik M, Papenfuß F, Port M, Bestvater F and Scherthan H: Super-resolution localization microscopy of radiation-induced histone $\mathrm{H} 2 \mathrm{AX}$-phosphorylation in relation to $\mathrm{H} 3 \mathrm{~K}$ 9-trimethylation in HeLa cells. Nanoscale 10: 4320-4331, 2018. 
35. Saint-André V, Batsché E, Rachez C and Muchardt C: Histone H3 lysine 9 trimethylation and HP1 $\gamma$ favor inclusion of alternative exons. Nat Struct Mol Biol 18: 337-344, 2011.

36. Bieberstein NI, Kozáková E, Huranová M, Thakur PK, Krchňáková Z, Krausová M, Carrillo Oesterreich F and Staněk D: TALE-directed local modulation of H3K9 methylation shapes exon recognition. Sci Rep 6: 29961, 2016.

37. Barrand S, Andersen IS and Collas P: Promoter-exon relationship of H3 lysine 9, 27, 30 and 79 methylation on pluripotency-associated genes. Biochem Biophys Res Commun 401: 611-617, 2010

38. Pedersen MT, Kooistra SM, Radzisheuskaya A, Laugesen A, Johansen JV, Hayward DG, Nilsson J, Agger K and Helin K: Continual removal of H3K9 promoter methylation by Jmjd2 demethylases is vital for ESC self-renewal and early development. EMBO J 35: 1550-1564, 2016.

39. Loh YH, Zhang W, Chen X, George J and Ng HH: Jmjd1a and Jmjd2c histone H3 Lys 9 demethylases regulate self-renewal in embryonic stem cells. Genes Dev 21: 2545-2557, 2007.

40. Vakoc CR, Mandat SA, Olenchock BA and Blobel GA: Histone $\mathrm{H} 3$ lysine 9 methylation and HP1gamma are associated with transcription elongation through mammalian chromatin. Mol Cell 19: 381-391, 2005.

41. Maksakova IA, Goyal P, Bullwinkel J, Brown JP, Bilenky M, Mager DL, Singh PB and Lorincz MC: H3K9me3-binding proteins are dispensable for SETDB1/H3K9me3-dependent retroviral silencing. Epigenetics Chromatin 4: 12, 2011.

42. Imai K, Kamio N, Cueno ME, Saito Y, Inoue H, Saito I and Ochiai K: Role of the histone H3 lysine 9 methyltransferase Suv39 h1 in maintaining Epsteinn-Barr virus latency in B95-8 cells. FEBS J 281: 2148-2158, 2014.

43. Lang F, Li X, Vladimirova O, Hu B, Chen G, Xiao Y, Singh V, Lu D, Li L, Han H, et al: CTCF interacts with the lytic HSV-1 genome to promote viral transcription. Sci Rep 7: 39861, 2017.

44. Fukuda A, Tomikawa J, Miura T, Hata K, Nakabayashi K, Eggan K, Akutsu H and Umezawa A: The role of maternal-specific $\mathrm{H} 3 \mathrm{~K} 9 \mathrm{me} 3$ modification in establishing imprinted X-chromosome inactivation and embryogenesis in mice. Nat Commun 5: 5464, 2014.

45. Mendelsohn AR and Larrick JW: Stem cell depletion by global disorganization of the $\mathrm{H} 3 \mathrm{~K} 9 \mathrm{me} 3$ epigenetic marker in aging. Rejuvenation Res 18: 371-375, 2015.
46. Koide S, Oshima M, Takubo K, Yamazaki S, Nitta E, Saraya A Aoyama K, Kato Y, Miyagi S, Nakajima-Takagi Y, et al: Setdb1 maintains hematopoietic stem and progenitor cells by restricting the ectopic activation of nonhematopoietic genes. Blood 128 638-649, 2016.

47. McDonald OG, Li X, Saunders T, Tryggvadottir R, Mentch SJ, Warmoes MO, Word AE, Carrer A, Salz TH, Natsume S, et al: Epigenomic reprogramming during pancreatic cancer progression links anabolic glucose metabolism to distant metastasis. Nat Genet 49: 367-376, 2017.

48. Chung YR, Schatoff E and Abdel-Wahab O: Epigenetic alterations in hematopoietic malignancies. Int J Hematol 96: 413-427, 2012.

49. Lehmann U, Brakensiek K and Kreipe H: Role of epigenetic changes in hematological malignancies. Ann Hematol 83: 137-152, 2004.

50. Becker JS, Nicetto D and Zaret KS: H3K9me3-dependent heterochromatin: Barrier to cell fate changes. Trends Genet 32: 29-41, 2016.

51. Wang C, Liu X, Gao Y, Yang L, Li C, Liu W, Chen C, Kou X, Zhao Y, Chen J, et al: Reprogramming of H3K9me3-dependent heterochromatin during mammalian embryo development. Nat Cell Biol 20: 620-631, 2018.

52. Nicetto D and Zaret KS: Role of H3K9me3 heterochromatin in cell identity establishment and maintenance. Curr Opin Genet Dev 55: 1-10, 2019

53. Adamkova K, Yi YJ, Petr J,Zalmanova T, Hoskova K, Jelinkova P, Moravec J, Kralickova M, Sutovsky M, Sutovsky P and Nevoral J: SIRT1-dependent modulation of methylation and acetylation of histone $\mathrm{H} 3$ on lysine 9 (H3K9) in the zygotic pronuclei improves porcine embryo development. J Anim Sci Biotechnol 8: 83, 2017.

54. Lee MG, Norman J, Shilatifard A and Shiekhattar R: Physical and functional association of a trimethyl H3K4 demethylase and Ring6a/MBLR, a polycomb-like protein. Cell 128: 877-887, 2017.

55. Li L and Wang Y: Cross-talk between the H3K36me 3 and H4K16ac histone epigenetic marks in DNA double-strand break repair. J Biol Chem 292: 11951-11959, 2017.

This work is licensed under a Creative Commons Attribution-NonCommercial-NoDerivatives 4.0 International (CC BY-NC-ND 4.0) License. 